\title{
A TOTAL LINEARIZATION METHOD FOR SOLVING VISCOUS FREE BOUNDARY FLOW PROBLEMS BY THE FINITE ELEMENT METHOD
}

\author{
N. P. KRUYT \\ Department of Mechanical Engineering, University of Twente, The Netherlands \\ C. CUVELIER AND A. SEGAL \\ Department of Mathematics and Informatics, Delft University of Technology, The Netherlands \\ AND \\ J. VAN DER ZANDEN \\ Department of Mechanical Engineering, Delft University of Technology, The Netherlands
}

\begin{abstract}
SUMMARY
In this paper a total linearization method is derived for solving steady viscous free boundary flow problems (including capillary effects) by the finite element method. It is shown that the influence of the geometrical unknown in the totally linearized weak formulation can be expressed in terms of boundary integrals. This means that the implementation of the method is simple. Numerical experiments show that the iterative method gives accurate results and converges very fast.
\end{abstract}

KEY WORDS Navier-Stokes equations Finite element method Viscous flow Free boundary flow

\section{INTRODUCTION}

In many problems in mechanics and physics the governing partial differential equations are defined on domains which are not known a priori. The boundaries of these domains are called free boundaries and must be determined as part of the solution. This means that the problem, apart from the usual unknown functions, contains additional geometrical unknowns.

A technologically important category of such free boundary problems is formed by the viscous free boundary flow problems, which occur, for example, in capillarity, coating and polymer technology. Because of the non-linear character of free boundary problems, almost all solutions to be found in the literature are of a numerical nature.

Concerning steady state free boundary problems, three basic approaches to the numerical solution by the finite element method can be distinguished in the literature:

(1) A fixed mesh is adopted and special techniques are developed to track the free boundary through the mesh. ${ }^{1}$ A disadvantage of this technique is that, in general, it is only possible to identify the elements in which the free boundary lies, but not its precise position. Furthermore, in order to follow the behaviour of the free boundary which undergoes even moderate changes of position, it is necessary to add numerical detail throughout a much larger part of the region than may actually be required.

0271-2091/88/030351-13\$06.50

(C) 1988 by John Wiley \& Sons, Ltd. 
(2) In the second approach ${ }^{2,3}$ a deforming spatial mesh is used and a three-stage iterative cycle is followed. First, a shape of the free boundary is assumed. Secondly, the boundary value problem on the resulting domain is solved after disregarding one of the boundary conditions at the free boundary. Thirdly, the shape of the free boundary is updated using the previously neglected boundary condition. This iterative cycle is repeated until some desired convergence criterion is satisfied. This approach suffers from a number of disadvantages. The iterative cycle often does not converge, and even when it does, being a fixed point type iteration, it converges slowly. Moreover, a new finite element problem must be solved at each iteration.

(3) The third, more recent approach ${ }^{4-8}$ also involves a deforming mesh but eliminates the successive iteration between the free boundary position and the field variables by introducing the position of the nodes at the free boundary directly as degrees of freedom. The non-linear equations are then solved using a Newton-Raphson (or a quasi-Newton ${ }^{9}$ ) iterative procedure which results in the simultaneous calculation of the position of the free boundary and the field variables. The advantage of this method is its second-order (or superlinear for the quasi-Newton method) rate of convergence. A disadvantage is that a complete account of the variations with respect to the free boundary degrees of freedom must be incorporated into the Jacobian of the system of equations. These variations, which involve integrals over a large part of the domain, have a non-local character. This means that the method does not fit into standard finite element codes, where the coefficient in the equations of an unknown belonging to a nodal point is determined completely by contributions over the neighbouring elements. This makes the implementation of the method relatively difficult. Another disadvantage is that a new finite element problem must be solved at each iteration.

The aim of this paper is to derive a numerical method, the total linearization method (TLM), which is much easier to implement than the Newton-Raphson algorithm while retaining its superior convergence properties. It is similar to References 10 and 11 , but in this paper it will be shown that the influence of the unknown position of the free boundary can be reduced completely to boundary integrals. This has great advantages for software implementation.

The sequel is restricted to the die-swell problem, which serves as a model case for the TLM. However, it is possible to apply the TLM to other free boundary problems as well.

In Section 2 the die-swell problem is described and its mathematical formulation is given. In Section 3 the weak formulation of the problem is derived. This weak formulation is linearized in Section 4 . The numerical method consists of a discretization of this linearized weak formulation. In Section 5 the numerical results are shown and compared with those available from the literature.

\section{MATHEMATICAL FORMULATION}

The extrusion of a viscous incompressible jet from a die into an inviscid fluid is of considerable rheological importance. It is observed that far downstream the height of the extrudate is different from that of the die. This phenomenon is known as die-swell or extrudate swell.

In this paper it is assumed that the jet is Newtonian and that the flow is steady and twodimensional in a domain $\Omega$ with boundary $\Gamma$ (see Figure 1). Furthermore it is assumed that there are no external forces.

The governing equations are

$$
\begin{aligned}
\rho u_{j} \frac{\partial}{\partial x_{j}} u_{i} & =\frac{\partial}{\partial x_{j}} T_{i j} & & \text { (momentum equations), } \\
\partial u_{i} / \partial x_{i} & =0 & & \text { (continuity equation), }
\end{aligned}
$$




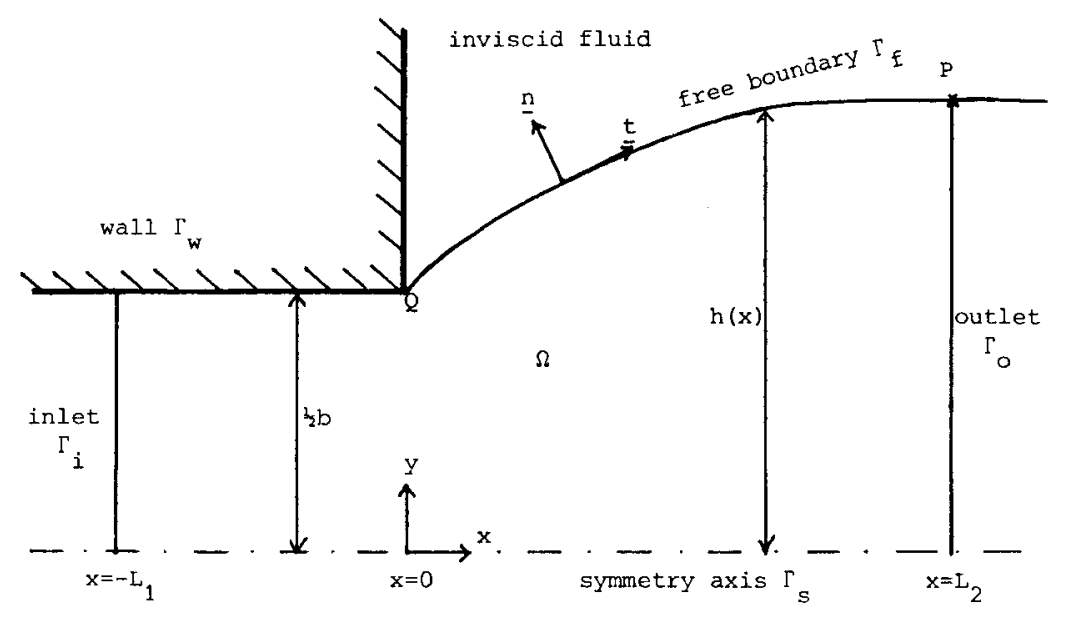

Figure 1. Geometry of the die-swell problem

where

$$
T_{i j}=-p \delta_{i j}+\mu\left(\frac{\partial u_{i}}{\partial x_{j}}+\frac{\partial u_{j}}{\partial x_{i}}\right) \quad \text { (constitutive equation) }
$$

is the stress tensor for a Newtonian fluid with dynamic viscosity $\mu$ and constant density $\rho$. The components of the velocity vector are $u_{i}$, the pressure is denoted by $p$ and $\delta_{i j}$ is the Kronecker symbol. At the inlet (width $b / 2$ ) the velocity distribution is a fully developed Poiseuille flow with mean velocity $U$. At the outlet it is supposed that the flow is parallel to the $x_{1}$ axis and that there is no diffusive outflow of momentum. At the wall the fluid is assumed to satisfy the no-slip condition. We also assume that the flow is symmetric with respect to the $\Gamma_{\mathrm{s}}$ boundary. Thus the boundary conditions are, apart from those at the free boundary,

at the inlet $\Gamma_{i}: u_{1}=\frac{3}{2} U\left[1-\left(2 x_{2} / b\right)^{2}\right], \quad u_{2}=0$,

at the outlet $\Gamma_{\mathrm{o}}: T_{i j} n_{j} n_{i}=0, \quad u_{i} t_{i}=0$,

at the wall $\Gamma_{\mathrm{w}}: u_{1}=0, \quad u_{2}=0$,

at the symmetry axis $\Gamma_{\mathrm{s}}: u_{i} n_{i}=0, \quad T_{i j} n_{j} t_{i}=0$,

where $n_{i}$ and $t_{i}$ are the components of the normal and tangential vector respectively on the boundary $\Gamma$. The orientation of the tangential vector is taken to be clockwise.

At the free boundary the conditions for a balance of forces must be fulfilled. These are the traction conditions for the normal and tangential components of the stress vector. Moreover, in the stationary situation the free boundary is a streamline, which leads to the kinematic condition for the velocity. When we assume that the pressure of the surrounding inviscid fluid equals zero, the boundary conditions at the free boundary $\Gamma_{\mathrm{f}}$ are

$$
\begin{aligned}
u_{i} n_{i} & =0 & & \text { (kinematic condition), } \\
T_{i j} n_{j} n_{i} & =\sigma / R & & \text { (normal stress condition), } \\
T_{i j} n_{j} t_{i} & =0 & & \text { (tangential stress condition), }
\end{aligned}
$$

where $\sigma$ is the coefficient of surface tension. The curvature $1 / R$ of the free boundary $(R>0$ at points 
of the free boundary where $\Omega$ is locally concave) is given by

$$
\frac{1}{R}=\frac{\mathrm{d}^{2} h}{\mathrm{~d} x_{1}^{2}} /\left[1+\left(\frac{\mathrm{d} h}{\mathrm{~d} x_{1}}\right)^{2}\right]^{3 / 2},
$$

where $h\left(x_{1}\right)$ is the distance between the $x_{1}$ axis and the free boundary. Note that the number of boundary conditions at the free boundary is equal to three instead of two, which would be the case for a fixed domain problem. This extra boundary condition is necessary because there is an additional geometrical unknown: the position of the free boundary.

Since the position of the free boundary is determined by the solution of a second-order nonlinear ordinary differential equation, two boundary conditions for the position of the free boundary are necessary. These boundary conditions are the vanishing slope at the outflow section and the fixed separation point at the wall:

at the outflow section: $\mathrm{d} h / \mathrm{d} x=0$ in $P$,

at the wall: $h=b / 2$ in $\mathrm{Q}$.

It is also possible to prescribe a different boundary condition at the wall; see, for example, References 12 and 13.

With the characteristic velocity $U$ and the characteristic length $b$, the following dimensionless groups can be formed:

the Reynolds number $R e$ defined by

$$
R e=\rho U b / \mu
$$

and the capillary number $\mathrm{Ca}$ defined by

$$
\mathrm{Ca}=\mu U / \sigma .
$$

Computations have been performed for realistic values of the Reynolds and the capillary number, namely $\mathrm{Re} \leqslant 300$ and $\mathrm{Ca}^{-1} \leqslant 4 \cdot 444$. For these ranges of the characteristic numbers no oscillatory solutions are observed.

\section{WEAK FORMULATION}

In order to solve the equations with the appropriate boundary conditions by the finite element method, a weak formulation of the problem must be derived. Multiplication of (1) by test functions $\phi=\left(\phi_{1}, \phi_{2}\right)^{\mathrm{T}}$ and integration by parts (Gauss's theorem) over the domain $\Omega$ results in the following weak formulation of the momentum equations:

$$
\rho \int_{\Omega} \phi_{i} u_{j} \frac{\partial}{\partial x_{j}} u_{i} \mathrm{~d} \Omega=\int_{\Gamma} T_{i j} n_{j} \phi_{i} \mathrm{~d} s-\int_{\Omega} T_{i j} \frac{\partial \phi_{i}}{\partial x_{j}} \mathrm{~d} \Omega
$$

for all test functions $\phi$ which are square integrable over $\Omega$ together with their first-order derivatives. The test functions $\phi$ must also be equal to zero at $\Gamma_{w} \cup \Gamma_{i}$ and must have vanishing tangential component at $\Gamma_{\mathrm{o}}$ and vanishing normal component at $\Gamma_{\mathrm{s}}$. Substitution of the boundary conditions (4) and (5b,c) gives:

$$
\rho \int_{\Omega} \phi_{i} u_{j} \frac{\partial}{\partial x_{j}} u_{i} \mathrm{~d} \Omega=\sigma \int_{\Gamma_{\mathrm{f}}} \phi_{i} n_{i} \frac{1}{R} \mathrm{~d} s-\int_{\Omega} T_{i j} \frac{\partial \phi_{i}}{\partial x_{j}} \mathrm{~d} \Omega .
$$

The order of the derivatives in the first term on the right-hand side of (11) can be reduced by the 
substitution

$$
\frac{1}{R} n_{i}=\frac{\mathrm{d} t_{i}}{\mathrm{~d} s}
$$

and by partial integration over $\Gamma_{\mathrm{f}}$. The result is, after substitution of the boundary conditions (7),

$$
\int_{\Gamma_{\mathrm{f}}} \phi_{i} n_{i} \frac{1}{R} \mathrm{~d} s=\delta_{i 1} \phi_{i}(\mathrm{P})-\int_{\Gamma_{\mathrm{f}}} t_{i} \frac{\mathrm{d} \phi_{i}}{\mathrm{~d} s} \mathrm{~d} s .
$$

Substitution of (13) into (11) yields the weak formulation of the momentum equations:

$$
\rho \int_{\Omega} \phi_{i} u_{j} \frac{\partial}{\partial x_{j}} u_{i} \mathrm{~d} \Omega=\sigma \delta_{i 1} \phi_{i}(\mathrm{P})-\sigma \int_{\Gamma_{\mathrm{f}}} t_{i} \frac{\mathrm{d} \phi_{i}}{\mathrm{~d} s} \mathrm{~d} s-\int_{\Omega} T_{i j} \frac{\partial \phi_{i}}{\partial x_{j}} \mathrm{~d} \Omega .
$$

The weak formulation of the continuity equation (2) reads

$$
\int_{\Omega} q \frac{\partial u_{i}}{\partial x_{i}} \mathrm{~d} \Omega=0
$$

for all test functions $q$ which are square integrable over $\Omega$.

The kinematic condition (5a) has not been imposed in the weak formulation (14). Its weak formulation is

$$
\int_{\Gamma_{\mathrm{f}}} \chi u_{i} n_{i} \mathrm{~d} s=0
$$

for all test functions $\chi$ which are square integrable over $\Gamma_{\mathrm{f}}$ as well as their first-order derivatives. In the sequel it will become clear why this choice has been made for the test functions $\chi$.

\section{LINEARIZATION OF THE WEAK FORMULATION}

Because free boundary problems are non-linear, the equations must be linearized with respect to the position of the free boundary, velocity and pressure. The first basic idea of the TLM is to linearize the continuous form of the weak formulation as was done previously. ${ }^{10,11,14}$ In this way the TLM may be considered as a variant of Newton's method applied to the continuous problem. This is in contrast to the classical method of Newton which performs a linearization of the discrete form of the free boundary problem. The second essential point of the TLM is to take account of the boundary conditions at the free boundary in order to simplify the linearization of the weak formulation. It will be shown that the influence of the free boundary can be reduced to boundary integrals.

Let $\Gamma_{\mathrm{f}}(h)$ be the position of the free boundary determined by the function $h(\mathbf{x})$ and let $\Gamma_{\mathrm{f}}\left(h_{0}\right)$ be an estimate of this position, corresponding to the function $h_{0}(\mathbf{x})$. The distance between $\Gamma_{\mathrm{f}}(h)$ and $\Gamma_{\mathrm{f}}\left(h_{0}\right)$ in the normal direction of $\Gamma_{\mathrm{f}}\left(h_{0}\right)$ is denoted by $\alpha(s)$ (see Figure 2 ).

Because of the essential boundary condition for the position of the free boundary given in $\mathrm{Q}$, we have

$$
\alpha(\mathrm{Q})=0 \text {. }
$$

By writing down the boundary conditions at $\Gamma_{0}$, it was assumed that the outlet $\Gamma_{0}$ is parallel to the $y$ axis. In order to keep the endpoint of $\Gamma_{\mathrm{f}}$ at $\Gamma_{\mathrm{o}}$, we take into account the segment $\mathrm{P}^{\prime} \mathrm{P}^{\prime \prime}$. $\mathrm{P}^{\prime \prime}$ is the point $\mathrm{P}$ replaced over a distance $\alpha(\mathrm{P})$ in the normal direction of $\Gamma_{\mathrm{f}}\left(h_{0}\right)$ and $\mathrm{P}^{\prime}$ is lying at $\Gamma_{0}$. The length of $\mathrm{P}^{\prime} \mathrm{P}^{\prime \prime}$ is denoted by $\beta(\mathrm{P})$ (see Figure 2 ). So we have

$$
\Gamma_{\mathrm{f}}(h)=\mathrm{QP}^{\prime} \quad \text { and } \quad \Gamma_{\mathrm{f}}\left(h_{0}\right)=\mathrm{QP} .
$$




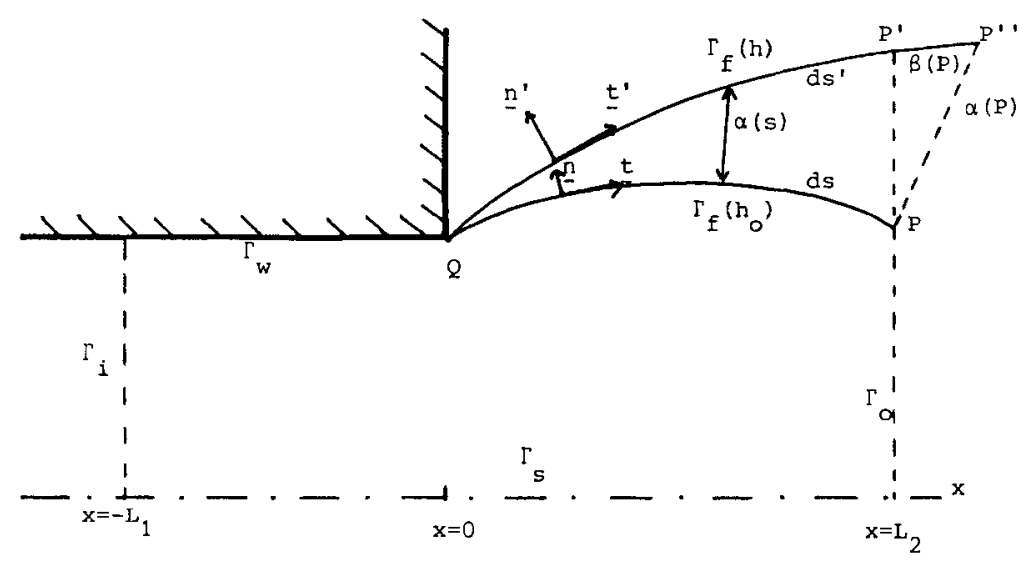

Figure 2. Free boundary $\Gamma_{\mathrm{f}}(h)$ and an approximation $\Gamma_{\mathrm{f}}\left(h_{0}\right)$

\subsection{Linearization of the surface integrals}

The linearization of the surface integrals with respect to the position of the free boundary is simple. In general we have

$$
\int_{\Omega(h)} f(\mathbf{x}) \mathrm{d} \Omega=\int_{\Omega\left(h_{0}\right)} f(\mathbf{x}) \mathrm{d} \Omega+\int_{\Gamma_{\mathbf{r}}\left(h_{0}\right)} \alpha f(s) \mathrm{d} s+o(\alpha)^{*},
$$

where $\Omega(h)$ and $\Omega\left(h_{0}\right)$ denote the domains determined by $\Gamma_{\mathrm{f}}(h)$ and $\Gamma_{\mathrm{f}}\left(h_{0}\right)$ respectively.

Using (19), the linearization of the convective terms in (14) with respect to the position of the free boundary becomes

$$
\rho \int_{\Omega(h)} \phi_{i} u_{j} \frac{\partial}{\partial x_{j}} u_{i} \mathrm{~d} \Omega=\rho \int_{\Omega\left(h_{0}\right)} \phi_{i} u_{j} \frac{\partial}{\partial x_{j}} u_{i} \mathrm{~d} \Omega+\rho \int_{\Gamma_{\mathrm{f}}\left(h_{0}\right)} \alpha \phi_{i} u_{j} \frac{\partial}{\partial x_{j}} u_{i} \mathrm{~d} s+o(\alpha) .
$$

Because of the kinematic condition (5a) at the free boundary, we have

$$
\left.u_{j} n_{j}\right|_{\Gamma_{\mathrm{r}}\left(h_{0}\right)}=\left.u_{j} n_{j}\right|_{\Gamma_{\mathrm{r}}(h)}+O(\alpha)=O(\alpha)
$$

This implies

$$
\begin{aligned}
\left.\left(u_{j} \frac{\partial}{\partial x_{j}} u_{i}\right)\right|_{\Gamma_{\mathrm{f}}\left(h_{0}\right)} & =\left.\left(\left(u_{k} n_{k}\right) n_{j} \frac{\partial}{\partial x_{j}} u_{i}+\left(u_{k} t_{k}\right) t_{j} \frac{\partial}{\partial x_{j}} u_{i}\right)\right|_{\Gamma_{\mathrm{f}}\left(h_{0}\right)} \\
& =\left.\left(u_{j} t_{j} \frac{\mathrm{d}}{\mathrm{d} s} u_{i}\right)\right|_{\Gamma_{\mathrm{r}}\left(h_{0}\right)}+O(\alpha) .
\end{aligned}
$$

Substitution of (22) into (20) reduces (20) to

$$
\rho \int_{\Omega(h)} \phi_{i} u_{j} \frac{\partial}{\partial x_{j}} u_{i} \mathrm{~d} \Omega=\rho \int_{\Omega\left(k_{0}\right)} \phi_{i} u_{j} \frac{\partial}{\partial x_{j}} u_{i} \mathrm{~d} \Omega+\rho \int_{\Gamma_{\mathrm{f}}\left(h_{0}\right)} \alpha u_{j} t_{j} \phi_{i} \frac{\mathrm{d}}{\mathrm{d} s} u_{i} \mathrm{~d} s+o(\alpha) .
$$

In a similar way the stress terms in (14) can be linearized with respect to the position of the free boundary. Using (19), this yields

$$
\int_{\Omega(h)} T_{i j} \frac{\partial \phi_{i}}{\partial x_{j}} \mathrm{~d} \Omega=\int_{\Omega\left(h_{0}\right)} T_{i j} \frac{\partial \phi_{i}}{\partial x_{j}} \mathrm{~d} \Omega+\int_{\Gamma_{\mathrm{r}}\left(h_{0}\right)} \alpha T_{i j} \frac{\partial \phi_{i}}{\partial x_{j}} \mathrm{~d} s+o(\alpha)
$$

* By $o(\alpha)$ we mean $o\left(\alpha, \mathrm{d} \alpha / \mathrm{d} s, \mathrm{~d}^{2} \alpha / \mathrm{d} s^{2}\right)$. The same applies to the $O(\alpha)$ symbol. 
In general we have

$$
\begin{aligned}
\left.T_{i j}\right|_{\Gamma_{\mathrm{r}}\left(h_{0}\right)}= & {\left.\left[\left(T_{k j} n_{j} n_{k}\right) n_{i} n_{j}+\left(T_{k j} n_{j} t_{k}\right) t_{i} n_{j}+\left(T_{k j} t_{j} n_{k}\right) n_{i} t_{j}\right]\right|_{\Gamma_{\mathrm{r}}(h)} } \\
& +\left.\left[\left(T_{k j} t_{j} t_{k}\right) t_{i} t_{j}\right]\right|_{\Gamma_{\mathrm{r}\left(h_{0}\right)}}+O(\alpha) .
\end{aligned}
$$

The traction conditions $(5 \mathrm{~b}, \mathrm{c})$ at the free boundary and the symmetry of the stress tensor imply that

$$
\begin{aligned}
\left.T_{i j}\right|_{\Gamma_{\mathbf{r}}\left(h_{0}\right)}= & {\left.\left[(\sigma / R) n_{i} n_{j}\right]\right|_{\Gamma_{\mathbf{f}(h)}}+\left.\left[\left(T_{k j} t_{j} t_{k}\right) t_{i} t_{j}\right]\right|_{\Gamma_{\mathbf{r}\left(h_{0}\right)}}+O(\alpha) . } \\
& =\left.\left[(\sigma / R) n_{i} n_{j}\right]\right|_{\Gamma_{\mathbf{r}\left(h_{0}\right)}}+\left.\left[\left(T_{k j} t_{j} t_{k}\right) t_{i} t_{j}\right]\right|_{\Gamma_{\mathbf{f}\left(h_{0}\right)}}+O(\alpha)
\end{aligned}
$$

Substitution of (26b) into (24) gives

$$
\begin{aligned}
\int_{\Omega(h)} T_{i j} \frac{\partial \phi_{i}}{\partial x_{j}} \mathrm{~d} \Omega= & \int_{\Omega\left(h_{0}\right)} T_{i j} \frac{\partial \phi_{i}}{\partial x_{j}} \mathrm{~d} \Omega+\sigma \int_{\Gamma_{\xi}\left(h_{0}\right)} \propto \frac{\partial \phi_{i}}{\partial x_{j}} n_{i} n_{j} \frac{1}{R} \mathrm{~d} s \\
& +\int_{\Gamma_{f}\left(h_{0}\right)} \alpha\left(-p+2 \mu t_{j} \frac{\mathrm{d} u_{j}}{\mathrm{~d} s}\right)_{t_{i}} \frac{\mathrm{d} \phi_{i}}{\mathrm{~d} s} \mathrm{~d} s+o(\alpha)
\end{aligned}
$$

where (3) has been used.

Using (19) and $\partial u_{i} / \partial x_{i}=O(\alpha)$ at $\Gamma_{\mathrm{f}}\left(h_{0}\right)$, the linearization of the continuity equation (15) with respect to the position of the free boundary becomes

$$
\int_{\Omega(h)} q \frac{\partial u_{i}}{\partial x_{i}} \mathrm{~d} \Omega=\int_{\Omega\left(h_{0}\right)} q \frac{\partial u_{i}}{\partial x_{i}} \mathrm{~d} \Omega+o(\alpha) .
$$

\subsection{Linearization of the kinematic condition and of the surface tension terms}

In order to linearize the boundary integrals in the weak formulation (14), (15) and (16), the following elementary relations are used:

$$
\begin{aligned}
\mathrm{d} s^{\prime} & =\left(1-\frac{\alpha}{R}\right) \mathrm{d} s+o(\alpha), \\
\frac{\mathrm{d}}{\mathrm{d} s^{\prime}} & =\left(1+\frac{\alpha}{R}\right) \frac{\mathrm{d}}{\mathrm{d} s}+o(\alpha), \\
\mathbf{n}^{\prime} & =\mathbf{n}-\frac{\mathrm{d} \alpha}{\mathrm{d} s} \mathbf{t}+o(\alpha), \\
\mathbf{t}^{\prime} & =\mathbf{t}+\frac{\mathrm{d} \alpha}{\mathrm{d} s} \mathbf{n}+o(\alpha) .
\end{aligned}
$$

For the notation we refer to Figure 2. Quantities without a prime denote quantities at $\Gamma_{\mathrm{f}}\left(h_{0}\right)$; primed quantities refer to $\Gamma_{\mathrm{f}}(h)$.

Using (29) and (31), the linearization of the kinematic condition (16) with respect to the position of the free boundary reads

$$
\int_{\Gamma_{\mathrm{r}}(h)} \chi^{\prime} u_{i}^{\prime} n_{i}^{\prime} \mathrm{d} s^{\prime}=\int_{\Gamma_{\mathrm{f}}\left(h_{0}\right)} \chi\left(u_{i}+\alpha n_{j} \frac{\partial u_{i}}{\partial x_{j}}\right)\left(n_{i}-\frac{\mathrm{d} \alpha}{\mathrm{d} s} t_{i}\right)\left(1-\frac{\alpha}{R}\right) \mathrm{d} s-\beta(\mathrm{P})\left(\chi u_{i} n_{i}\right)(\mathrm{P})+o(\alpha)
$$

In (33) the relation $\chi^{\prime}=\chi$ is used because the test functions $\chi$ for the position of the free boundary 
are defined at $\Gamma_{\mathrm{f}}$ only. With (12), rearranging (33) leads to

$$
\begin{aligned}
\int_{\Gamma_{\mathrm{f}}(h)} \chi^{\prime} u_{i}^{\prime} n_{i}^{\prime} \mathrm{d} s^{\prime}= & \int_{\Gamma_{\mathrm{f}}\left(h_{0}\right)} \chi u_{i} n_{i} \mathrm{~d} s+\int_{\Gamma_{\mathrm{f}}\left(h_{0}\right)} \chi \alpha \frac{\partial u_{i}}{\partial x_{j}} n_{i} n_{j} \mathrm{~d} s \\
& -\int_{\Gamma_{\mathrm{r}\left(h_{0}\right)}} \chi u_{i} \frac{\mathrm{d}}{\mathrm{d} s}\left(\alpha t_{i}\right) \mathrm{d} s-\beta(\mathrm{P})\left(\chi u_{i} n_{i}\right)(\mathrm{P})+o(\alpha) .
\end{aligned}
$$

Partial integration of the third term on the right-hand side and substitution of (17) yields

$$
\begin{aligned}
& \int_{\Gamma_{\mathrm{f}}(h)} \chi^{\prime} u_{i}^{\prime} n_{i}^{\prime} \mathrm{d} s^{\prime}=\int_{\Gamma_{\mathrm{r}}\left(h_{0}\right)} \chi u_{i} n_{i} \mathrm{~d} s+\int_{\Gamma_{\mathrm{f}}\left(h_{0}\right)} \chi \alpha \frac{\partial u_{i}}{\partial x_{j}}\left(n_{i} n_{j}+t_{i} t_{j}\right) \mathrm{d} s \\
& \quad+\int_{\Gamma_{\mathrm{r}}\left(h_{0}\right)} \alpha u_{i} t_{i} \frac{\mathrm{d} \chi}{\mathrm{d} s} \mathrm{~d} s-\alpha(\mathrm{P})\left(\chi u_{i} t_{i}\right)(\mathrm{P})-\beta(\mathrm{P})\left(\chi u_{i} n_{i}\right)(\mathrm{P})+o(\alpha) .
\end{aligned}
$$

Because $n_{i} n_{j}+t_{i} t_{j}=\delta_{i j}, \partial u_{i} / \partial x_{i}=O(\alpha)$ and $u_{i} n_{i}=O(\alpha)$ at $\Gamma_{\mathrm{f}}\left(h_{0}\right)$, (35) can be simplified to

$$
\int_{\Gamma_{\mathrm{f}(h)}} \chi^{\prime} u_{i}^{\prime} n_{i}^{\prime} \mathrm{d} s^{\prime}=\int_{\Gamma_{\mathrm{f}}\left(h_{0}\right)} \chi u_{i} n_{i} \mathrm{~d} s+\int_{\Gamma_{\mathrm{f}}\left(h_{0}\right)} \alpha u_{i} t_{i} \frac{\mathrm{d} \chi}{\mathrm{d} s} \mathrm{~d} s-\alpha(\mathrm{P})\left(\chi u_{i} t_{i}\right)(\mathrm{P})+o(\alpha) .
$$

Because of the boundary condition (7a), we have

$$
t_{i}(\mathrm{P})=\delta_{i 1}+O(\alpha)
$$

Substitution of (37) into (36) gives

$$
\int_{\Gamma_{\mathrm{r}(h)}} \chi^{\prime} u_{i}^{\prime} n_{i}^{\prime} \mathrm{d} s^{\prime}=\int_{\Gamma_{\mathbf{f}}\left(h_{0}\right)} \chi u_{i} n_{i} \mathrm{~d} s+\int_{\Gamma_{\mathrm{r}}\left(h_{0}\right)} \alpha u_{i} t_{i} \frac{\mathrm{d} \chi}{\mathrm{d} s} \mathrm{~d} s-\alpha(\mathrm{P})\left(\chi u_{i} \delta_{i 1}\right)(\mathrm{P})+o(\alpha) .
$$

Note that in the derivation of (38) it has been assumed that the test functions $\chi$ have square integrable first-order derivatives.

Using (29), (30) and (32), the linearization of the term

$$
\int_{\Gamma_{\mathrm{r}}(h)} t_{i}^{\prime} \frac{\mathrm{d} \phi_{i}^{\prime}}{\mathrm{d} s^{\prime}} \mathrm{d} s^{\prime}
$$

in (3.5) becomes

$$
\begin{aligned}
\int_{\Gamma_{\mathbf{f}}(h)} t_{i}^{\prime} \frac{\mathrm{d}}{\mathrm{d} s^{\prime}} \phi_{i}^{\prime} \mathrm{d} s^{\prime}= & \int_{\Gamma_{\mathbf{f}}\left(h_{0}\right)}\left(t_{i}+\frac{\mathrm{d} \alpha}{\mathrm{d} s} n_{i}\right)\left(1+\frac{\alpha}{R}\right) \frac{\mathrm{d}}{\mathrm{d} s}\left(\phi_{i}+\alpha \frac{\partial \phi_{i}}{\partial n}\right)\left(1-\frac{\alpha}{R}\right) \mathrm{d} s \\
& -\beta(\mathrm{P})\left(t_{i} \frac{\mathrm{d} \phi_{i}}{\mathrm{~d} s}\right)(\mathrm{P})+o(\alpha) .
\end{aligned}
$$

Rearranging (39) yields

$$
\begin{aligned}
\int_{\Gamma_{\mathrm{f}}(h)} t_{i}^{\prime} \frac{\mathrm{d}}{\mathrm{d} s^{\prime}} \phi_{i}^{\prime} \mathrm{d} s^{\prime}= & \int_{\Gamma_{f}\left(h_{0}\right)} t_{i} \frac{\mathrm{d} \phi_{i}}{\mathrm{~d} s} \mathrm{~d} s+\int_{\Gamma_{\mathrm{r}}\left(h_{0}\right)} t_{i} \frac{\mathrm{d}}{\mathrm{d} s}\left(\alpha \frac{\partial \phi_{i}}{\partial n}\right) \mathrm{d} s \\
& +\int_{\Gamma_{\mathrm{f}}\left(h_{0}\right)} n_{i} \frac{\mathrm{d} \phi_{i}}{\mathrm{~d} s} \frac{\mathrm{d} \alpha}{\mathrm{d} s} \mathrm{~d} s-\beta(\mathrm{P})\left(t_{i} \frac{\mathrm{d} \phi_{i}}{\mathrm{~d} s}\right)(\mathrm{P})+o(\alpha)
\end{aligned}
$$


Partial integration of the second term on the right-hand side and substitution of (17) and (12) gives

$$
\begin{aligned}
\int_{\Gamma_{\mathrm{f}}(h)} t_{i}^{\prime} \frac{\mathrm{d}}{\mathrm{d} s^{\prime}} \phi_{i}^{\prime} \mathrm{d} s^{\prime}= & \int_{\Gamma_{\mathrm{f}}\left(h_{0}\right)} t_{i} \frac{\mathrm{d} \phi_{i}}{\mathrm{~d} s} \mathrm{~d} s+\int_{\Gamma_{\mathrm{f}}\left(h_{0}\right)} n_{i} \frac{\mathrm{d} \phi_{i}}{\mathrm{~d} s} \frac{\mathrm{d} \alpha}{\mathrm{d} s} \mathrm{~d} s \\
& -\int_{\Gamma_{\mathrm{f}\left(h_{0}\right)}} \alpha \frac{\partial \phi_{i}}{\partial x_{j}} n_{i} n_{j} \frac{1}{R} \mathrm{~d} s+\alpha(\mathrm{P})\left(t_{i} \frac{\partial \phi_{i}}{\partial n}\right)(\mathrm{P})-\beta(\mathrm{P})\left(t_{i} \frac{\mathrm{d} \phi_{i}}{\mathrm{~d} s}\right)(\mathrm{P})+o(\alpha) .
\end{aligned}
$$

The extrapolation of the term $\delta_{i 1} \phi_{i}^{\prime}\left(\mathbf{P}^{\prime}\right)$ in (14) reads

$$
\delta_{i 1} \phi_{i}^{\prime}\left(\mathrm{P}^{\prime}\right)=\delta_{i 1}\left(\phi_{i}(\mathrm{P})+\alpha(\mathrm{P}) \frac{\partial \phi_{i}}{\partial n}(\mathrm{P})-\beta(\mathrm{P}) \frac{\mathrm{d} \phi_{i}}{\mathrm{~d} s}(\mathrm{P})\right)+o(\alpha) .
$$

The relations (37), (41) and (42) lead to the following linearization of the surface tension terms with respect to the position of the free boundary:

$$
\begin{gathered}
\sigma \delta_{i 1} \phi_{i}^{\prime}\left(\mathrm{P}^{\prime}\right)-\sigma \int_{\Gamma_{\mathrm{r}}(h)} t_{i}^{\prime} \frac{\mathrm{d}}{\mathrm{d} s^{\prime}} \phi_{i}^{\prime} \mathrm{d} s^{\prime}=\sigma \delta_{i 1} \phi_{i}(\mathrm{P})-\sigma \int_{\Gamma_{\mathrm{f}}\left(h_{0}\right)} t_{i} \frac{\mathrm{d} \phi_{i}}{\mathrm{~d} s} \mathrm{~d} s \\
-\sigma \int_{\Gamma_{\mathrm{r}\left(h_{0}\right)}} n_{i} \frac{\mathrm{d} \phi_{i}}{\mathrm{~d} s} \frac{\mathrm{d} \alpha}{\mathrm{d} s} \mathrm{~d} s+\sigma \int_{\Gamma_{\mathrm{f}}\left(h_{0}\right)} \alpha \frac{\partial \phi_{i}}{\partial x_{j}} n_{i} n_{j} \frac{1}{R} \mathrm{~d} s+o(\alpha) .
\end{gathered}
$$

\subsection{The total linearization of the free boundary problem}

Using (23), (27) and (43) the linearization of the momentum equations (14) with respect to the position of the free boundary can be written as

$$
\begin{aligned}
& \rho \int_{\Omega\left(h_{0}\right)} \phi_{i} u_{j} \frac{\partial}{\partial x_{j}} u_{i} \mathrm{~d} \Omega+\rho \int_{\Gamma_{\mathrm{f}}\left(h_{0}\right)} \alpha \phi_{i} u_{j} t_{j} \frac{\mathrm{d} u_{i}}{\mathrm{~d} s} \mathrm{~d} s+\int_{\Omega\left(h_{0}\right)} T_{i j} \frac{\partial \phi_{i}}{\partial x_{j}} \mathrm{~d} \Omega \\
& \quad+\int_{\Gamma_{\mathrm{f}}\left(h_{0}\right)} \alpha\left(-p+2 \mu t_{j} \frac{\partial u_{j}}{\mathrm{~d} s}\right) t_{i} \frac{\mathrm{d} \phi_{i}}{\mathrm{~d} s} \mathrm{~d} s+\sigma \int_{\Gamma_{\mathrm{f}}\left(h_{0}\right)} n_{i} \frac{\mathrm{d} \phi_{i}}{\mathrm{~d} s} \frac{\mathrm{d} \alpha}{\mathrm{d} s} \mathrm{~d} s \\
& =\sigma \delta_{i 1} \phi_{i}(\mathrm{P})-\sigma \int_{\Gamma_{\mathrm{f}\left(h_{0}\right)}} t_{i} \frac{\mathrm{d} \phi_{i}}{\mathrm{~d} s} \mathrm{~d} s+o(\alpha) .
\end{aligned}
$$

In (44) a linearization with respect to the unknowns $u_{i}$ and $p$ has not yet been carried out. The convective terms are linearized as described in Reference 15 . When higher-order terms are neglected, the total linearization of the momentum equations becomes

$$
\begin{aligned}
& \rho \int_{\Omega\left(h_{0}\right)} \phi_{i}\left(u_{j} \frac{\partial}{\partial x_{j}} u_{i}^{0}+u_{j}^{0} \frac{\partial}{\partial x_{j}} u_{i}\right) \mathrm{d} \Omega+\rho \int_{\Gamma_{\mathrm{r}\left(h_{0}\right)}} \alpha u_{j}^{0} t_{j} \phi_{i} \frac{\mathrm{d} u_{i}^{0}}{\mathrm{~d} s} \mathrm{~d} s \\
& \quad+\int_{\Omega\left(h_{0}\right)} T_{i j} \frac{\partial \phi_{i}}{\partial x_{j}} \mathrm{~d} \Omega+\int_{\Gamma_{\mathrm{f}}\left(h_{0}\right)} \alpha\left(-p^{0}+2 \mu t_{j} \frac{\mathrm{d} u_{j}^{0}}{\mathrm{~d} s}\right) t_{i} \frac{\mathrm{d} \phi_{i}}{\mathrm{~d} s} \mathrm{~d} s+\sigma \int_{\Gamma_{\mathrm{f}}\left(h_{0}\right)} n_{i} \frac{\mathrm{d} \phi_{i}}{\mathrm{~d} s} \frac{\mathrm{d} \alpha}{\mathrm{d} s} \mathrm{~d} s \\
& =\rho \int_{\Omega_{\left(h_{0}\right)}} \phi_{i} u_{j}^{0} \frac{\partial}{\partial x_{j}} u_{i}^{0} \mathrm{~d} \Omega+\sigma \delta_{i 1} \phi_{i}(\mathrm{P})-\sigma \int_{\Gamma_{\mathrm{f}}\left(h_{0}\right)} t_{i} \frac{\mathrm{d} \phi_{i}}{\mathrm{~d} s} \mathrm{~d} s,
\end{aligned}
$$

where

$$
u_{i}=u_{i}^{0}+\delta u_{i} \text { and } p=p^{0}+\delta p
$$

$u^{0}$ and $p^{0}$ are estimates of the velocity and pressure respectively.

When higher-order terms are neglected, the totally linearized continuity equation (28) is 


$$
\int_{\Omega\left(h_{0}\right)} q \frac{\partial u_{i}}{\partial x_{i}} \mathrm{~d} \Omega=0
$$

and the total linearization of the kinematic condition (38) reads

$$
\int_{\Gamma_{\mathbf{r}}\left(h_{0}\right)} \chi u_{i} n_{i} \mathrm{~d} s+\int_{\Gamma_{\mathrm{f}}\left(h_{0}\right)} \alpha u_{i}^{0} t_{i} \frac{\mathrm{d} \chi}{\mathrm{d} s} \mathrm{~d} s-\alpha(\mathrm{P}) \delta_{i 1}\left(\chi u_{i}^{0}\right)(\mathrm{P})=0 .
$$

Note that in the totally linearized weak formulation (45), (46) and (47) the influence of the geometrical unknown $\alpha$ is restricted to the free boundary. This means that the terms associated with $\alpha$ can be evaluated using boundary integrals.

\subsection{Algorithm}

The totally linearized weak formulation (45), (46) and (47) is discretized using a (triangular) finite element method of standard Galerkin type. The test functions $\phi, q$ and $\chi$ are replaced by

$$
\boldsymbol{\phi}=\left(\begin{array}{c}
\phi^{i} \\
0
\end{array}\right), \quad \boldsymbol{\phi}=\left(\begin{array}{c}
0 \\
\phi^{i}
\end{array}\right), \quad q=q^{i}, \quad \chi=\chi^{i},
$$

where $\phi^{i}, q^{i}$ and $\chi^{i}$ are the basis functions for the velocity, the pressure and the position of the free boundary respectively. The unknowns are written as

$$
u_{i}=u_{i}^{j} \phi^{j}, \quad \quad p=p^{j} q^{j}, \quad \alpha=\alpha^{j} \chi^{j} .
$$

The velocity is approximated by continuous extended quadratic polynomials. The pressure is approximated by piecewise linear polynomials which are not necessarily continuous. The geometrical unknown $\alpha$ is approximated by continuous quadratic one-dimensional polynomials. Finally, the discretized continuity equation is replaced by a penalty term. For a complete description of these techniques, we refer to Reference 15.

The iterative numerical methods consists of the following steps:

Estimate $\Gamma_{\mathrm{f}}\left(h^{0}\right), u_{i}^{0}$ and $p^{0} ; \alpha^{0}=0$.

$u_{i}^{0}$ and $p^{0}$ may be found by solving the Stokes equations on the fixed domain.

For $k:=0, k+1$ while $\left|\alpha^{k+1}\right|>\varepsilon$ or $\left\|\mathbf{u}^{k+1}-\mathbf{u}^{k}\right\|>\varepsilon$ do:

Solve $u_{i}^{k+1}, p^{k+1}$ and $\alpha^{k+1}$ by solving the discretized form of the equations (45), (46) and (47). A penalty method may be applied for the elimination of the pressure

Update the free boundary by

$$
\Gamma_{\mathrm{f}}\left(h^{k+1}\right)=\Gamma_{\mathrm{f}}\left(h^{k}\right)+\alpha^{k+1} \mathbf{n}
$$

where $\mathbf{n}$ is the discrete normal on $\Gamma_{\mathrm{f}}$. At the endpoints of elements the average value of $\mathbf{n}$ between two boundary elements is used.

Generate a new mesh using the updated free boundary $\Gamma_{\mathrm{f}}\left(h^{k+1}\right)$.

The iteration process may be accelerated by correcting the nodal values at the new mesh by interpolating from the old ones. However, such a step requires extra software for the interpolation problem.

The solution of $u_{i}^{k+1}, p^{k+1}$ and $\alpha^{k+1}$ is easily implemented, because it is equivalent to a standard Navier-Stokes formulation for the velocity and pressure, supplemented with boundary integrals which involve the additional geometrical unknown $\alpha$. 
The expected rate of convergence of the TLM is at least superlinear, because the equations have been linearized correctly.

A major advantage of the TLM over the method of Newton-Raphson is the restriction of the influence of the geometrical unknown to the free boundary. This causes a reduction of the profile width of the large matrix and of the costs associated with the assembly of the large matrix. This means that the CPU time required for one iteration decreases. The most important advantage of the TLM, however, lies in the ease of implementation.

\section{NUMERICAL RESULTS}

At each iteration the resulting system of linear equations is solved by a profile method.

The following initial estimates are used:

$$
u_{1}^{0} \equiv U, \quad u_{2}^{0} \equiv 0, \quad p^{0} \equiv 0, \quad h^{0} \equiv b / 2 .
$$

Convergence of the iterative process is determined in terms of the difference between two successive approximations of the velocity and of the distance between two successive approximations of the position of the free boundary. The iterative process is said to have converged when

$$
\left\|\mathbf{u}^{(k+1)}-\mathbf{u}^{(k)}\right\|_{\infty}<10^{-3} \quad \text { and } \quad\left\|\boldsymbol{\alpha}^{(k+1)}\right\|_{\infty}<10^{-3},
$$

where $\|\cdot\|_{\infty}$ denotes the maximum norm in the discrete space.

A series of simulations for different values of $\mathrm{Re}$ and $\mathrm{Ca}$ is performed. The results are compared with those of Engelman, ${ }^{8}$ Dupret $^{11}$ and Omodei ${ }^{3}$ in Table I. The parameter of practical interest is the die-swell percentage $r_{\mathrm{s}}$, defined by

$$
r_{\mathrm{s}}=100\left[h\left(L_{2}\right)-h(0)\right] / h(0) \text {. }
$$

The present results are obtained with a mesh consisting of 309 elements (including 15 line elements), a total of 1290 velocity unknowns and 31 free boundary unknowns. No interpolation (step 4) has been carried out. Figures 3 and 4 show the streamlines for $R e=1, C a^{-1}=0.4$ and

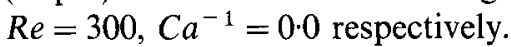

When $C a^{-1}=0.0$ it can be shown that in the first iteration, owing to the initial estimates (50), a zero appears on the main diagonal of the large matrix. The results in Table I with $C a^{-1}=0.0$ are

\begin{tabular}{|c|c|c|c|c|c|c|c|}
\hline \multirow{2}{*}{$R e$} & \multirow{2}{*}{$C a^{-1}$} & \multicolumn{2}{|c|}{ Present method } & \multirow{2}{*}{$\frac{\text { Engelman }}{r_{\mathrm{s}}}$} & \multirow{2}{*}{$\frac{\text { Dupret }}{r_{\mathrm{s}}}$} & \multicolumn{2}{|c|}{ Omodei } \\
\hline & & $\begin{array}{l}\text { Number of } \\
\text { iterations }\end{array}$ & $r_{\mathrm{s}}$ & & & $r_{\mathrm{s}}$ & \\
\hline 0.0 & $0 \cdot 0$ & 5 & $19 \cdot 28$ & - & 19.56 & $19 \cdot 0$ & \\
\hline \multirow[t]{4}{*}{$1 \cdot 0$} & 0.0 & 4 & $19 \cdot 17$ & 18.97 & 19.59 & $19 \cdot 1$ & \\
\hline & $0 \cdot 4$ & 4 & 16.88 & $16 \cdot 67$ & $16 \cdot 92$ & $16 \cdot 6$ & \\
\hline & 1.6 & 4 & $11 \cdot 76$ & 11.58 & 11.65 & $11 \cdot 4$ & \\
\hline & $3 \cdot 6$ & 4 & $7 \cdot 50$ & $7 \cdot 3$ & $7 \cdot 41$ & $6 \cdot 4$ & $( \pm \mathrm{osc})$ \\
\hline \multirow[t]{3}{*}{$4 \cdot 0$} & 0.4 & 4 & $15 \cdot 49$ & 15.47 & $15 \cdot 69$ & $15 \cdot 5$ & \\
\hline & 1.6 & 4 & 12.44 & $12 \cdot 34$ & 12.41 & $12 \cdot 2$ & \\
\hline & $2 \cdot 4$ & 4 & $10 \cdot 67$ & 10.57 & $10 \cdot 62$ & $10-4$ & $( \pm$ osc $)$ \\
\hline \multirow[t]{2}{*}{$18 \cdot 0$} & $2 \cdot 222$ & 5 & 1.25 & $1 \cdot 31$ & $1 \cdot 35$ & $1 \cdot 3$ & $( \pm$ osc $)$ \\
\hline & 4.444 & 5 & 1.95 & $2 \cdot 00$ & 2.02 & $2 \cdot 2$ & (土osc) \\
\hline $75 \cdot 0$ & $2 \cdot 0$ & 6 & $-11 \cdot 21$ & $-11 \cdot 16$ & $-10 \cdot 92$ & $-10 \cdot 48$ & $( \pm$ osc $)$ \\
\hline $300-0$ & 0.0 & 6 & $-15 \cdot 24$ & $-15 \cdot 24$ & - & -15.52 & \\
\hline
\end{tabular}
obtained by using a value of $10^{-3}$ for $\mathrm{Ca}^{-1}$ in the first iteration.

Table I 


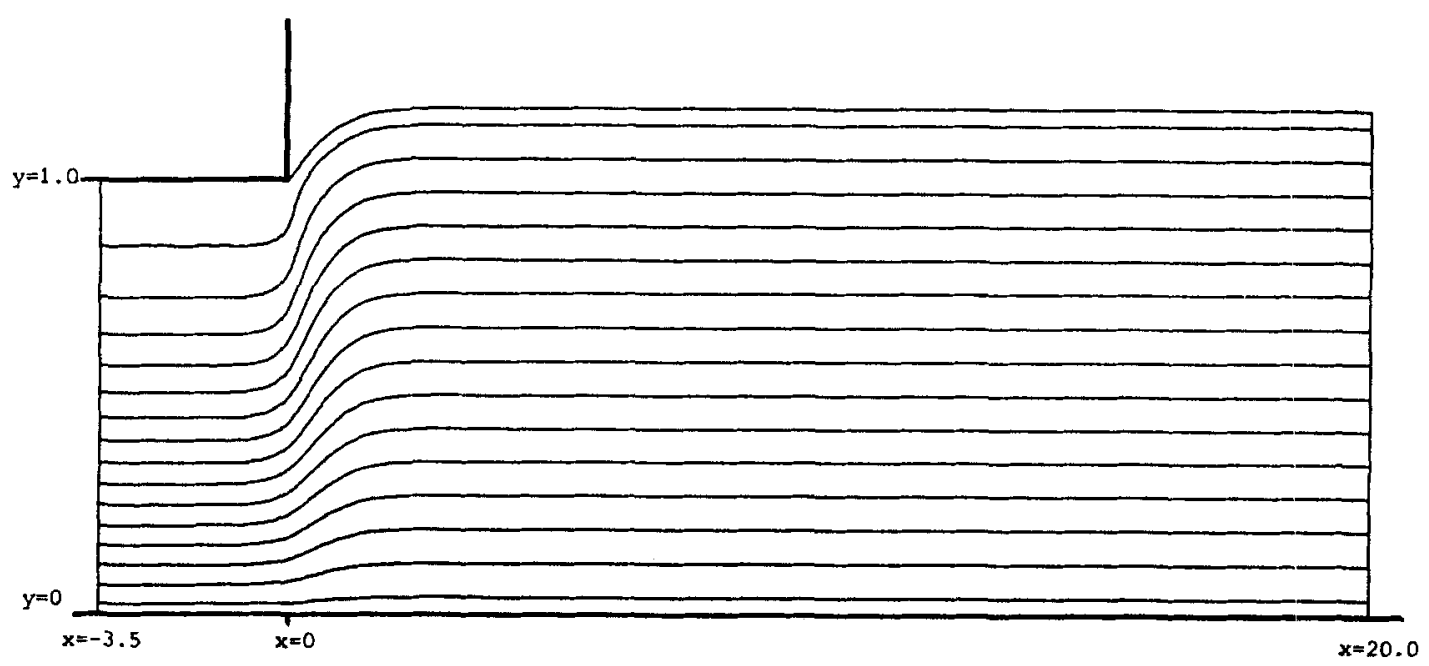

Figure 3. $R e=1 \cdot 0, C a^{-1}=0.4$

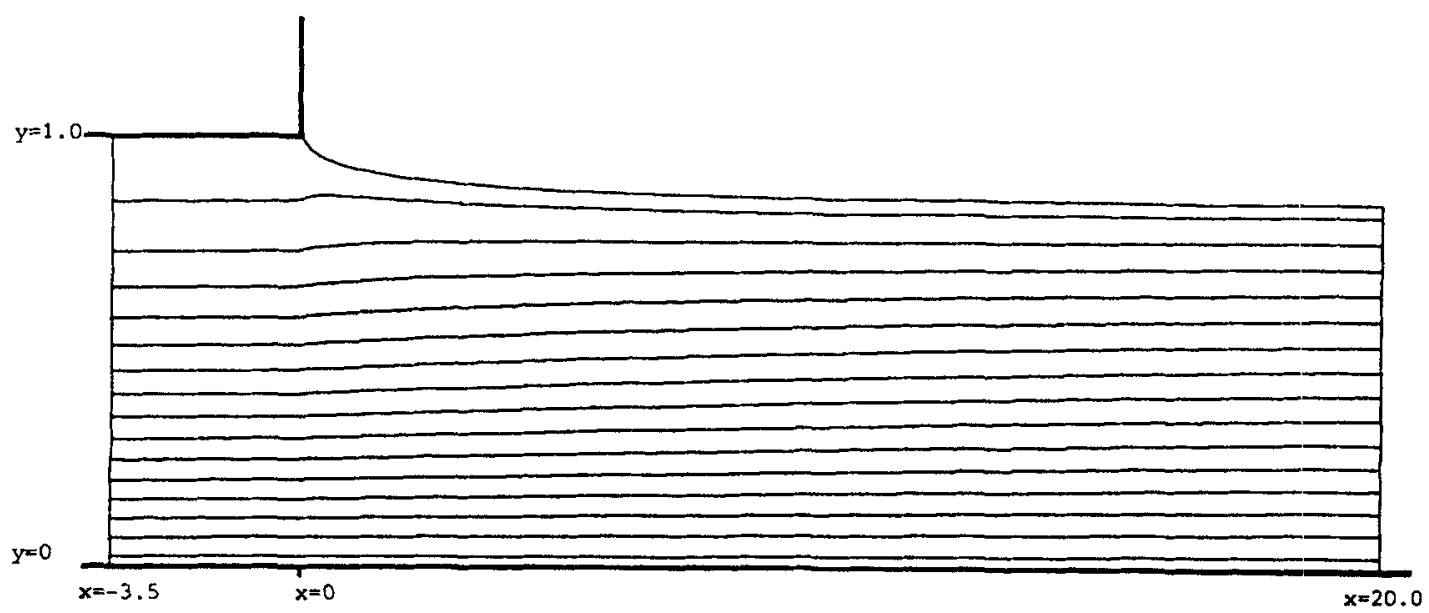

Figure 4. $\mathrm{Re}=300, \mathrm{Ca}^{-1}=0 \cdot 0$

In order to verify the mesh dependence of the convergence of the iterative procedure, we have run the same problem for $\mathrm{Re}=1$ and $\mathrm{Ca}^{-1}=0.4$ on three different meshes:

mesh 1: 96 elements, 370 velocity unknowns and 15 free boundary unknowns mesh 2: 368 elements, 1444 velocity unknowns and 31 free boundary unknowns mesh 3: 1440 elements, 5704 velocity unknowns and 63 free boundary unknowns.

Meshes 1 and 2 both took five iterations to get $\left\|\alpha^{k+1}\right\|<10^{-3}$; mesh 3 only needed four iterations. Clearly, the speed of convergence of the method does not depend on the mesh size; on the contrary, the finest mesh with a smooth solution required the least number of iterations.

As can be seen from Table I, our results are in close agreement with those of Engelman, Dupret and Omodei. The oscillations reported in the results of Omodei are absent in our results. It is hardly possible to compare the required number of iterations, because Engelman and Dupret used the solution from a previous run as the initial solution vector. Using such initial estimates, 
Engelman required two or three iterations and Dupret required six or seven iterations to obtain convergence.

Owing to the presence of some fluctuations in the convergence factor, it is not clear whether the rate of convergence of the TLM is linear or superlinear. However, the iteration process always converges very fast, as can be seen from Table I. A possible cause of the fluctuations lies in the presence of discretization errors. Owing to these errors, the ultimate discrete solution will not satisfy exactly the boundary conditions at the free boundary which have been used in the derivation of the numerical method. This may result in a smaller rate of convergence.

A last experiment is performed in order to investigate the effect of interpolation (step 4). When $R e=0.0$ (Stokes flow) and $C a^{-1}=0.0$ the required number of iterations is four when using interpolation and five when not using interpolation. This indicates that the interpolation increases the rate of convergence significantly.

\section{CONCLUSION}

In this paper we have derived the total linearization method, a numerical technique for solving viscous free boundary flow problems by the finite element method. The numerical experiments show that this iterative method gives accurate results and converges very fast. The main advantage of the TLM over the Newton--Raphson algorithm lies in the ease of implementation, while it is very competitive with regard to the required number of iterations. Although we have restricted our attention to viscous free boundary flow problems, it seems that the TLM can be applied to general free boundary problems.

\section{ACKNOWLEDGEMENT}

This work was performed while the first author was receiving a grant from the Delft University Fund (Delfts Hogeschool Fonds).

\section{REFERENCES}

1. K. J. Bathe and M. R. Khoshgoftaar, 'Finite element free surface seepage analysis without mesh iteration', Int. j. numer. methods geomech., 3, 13-22 (1979).

2. W. J. Silliman and L. E. Scriven, 'Separating flow near a static contact-line: slip at a wall and shape of a free surface', $J$. Comput. Phys., 34, 287-313 (1980).

3. B. J. Omodei, 'Computer solutions of a plane Newtonian jet with surface tension', Comput. Fluids, 7, 79-96 (1979).

4. K. J. Ruschak, 'A method for incorporating free boundaries with surface tension in finite element fluid-flow simulators', Int. j. numer. methods eng., 15, 639-648 (1980).

5. H. Saito and L. E. Scriven, 'Study of coating flow by the finite element method', J. Comput. Phys., 42, 53-76 (1982).

6. H. M. Ettouney and R. A. Brown, 'Finite element methods for steady solidification problems', J. Comput. Phys., 49, $118-150$ (1983).

7. S. F. Kistler and L. E. Scriven, "Coating flow theory by finite element and asymptotic analysis of the Navier-Stokes system', Int. j. numer. methods fluids, 4, 207-229 (1984).

8. M. S. Engelman, FIDAP Theoretical Manual, 1984.

9. J. E. Dennis and J. J. More, 'Quasi-Newton methods, motivation and theory', SIAM Rev., 19, 46-89 (1977).

10. F. Dupret, 'Etude numérique d'écoulements irrotationnels incompressibles avec tension superficielle par une méthode variationelle', J. Mécan., 20, 659-690 (1981).

11. F. Dupret, 'A method for the computation of viscous flow by finite elements with free boundaries and surface tension', in T. Kawai (ed.), Finite Element Flow Analysis, University of Tokyo Press, Tokyo, 1982.

12. S. H. Davis, 'Contact-line problems in fluid mechanics', J. Appl. Mech., 50, 977-982 (1983).

13. R. I. Tanner, H. Lam and M. B. Bush, 'The separation of viscous jets', Phys. Fluids, 28, 23-25 (1985).

14. O. Pironneau, 'Optimal shape design for elliptic systems', Springer Series in Computational Physics, 1983.

15. C. Cuvelier, A. Segal and A. A. Van Steenhoven, Finite-Element Methods and Navier-Stokes equations, Reidel Publishing Company, Dordrecht, 1986. 